\title{
The evolution of the Milky Way from its earliest phases: Constraints on stellar nucleosynthesis
}

\author{
P. François ${ }^{1,2}$, F. Matteucci ${ }^{3}$, R. Cayrel ${ }^{1}$, M. Spite ${ }^{4}$, F. Spite ${ }^{4}$, and C. Chiappini ${ }^{5,2}$ \\ 1 Observatoire de Paris/Meudon, GEPI, 61 avenue de l'Observatoire, 75014 Paris, France \\ 2 Visiting scientist at European Southern Observatory, Karl Schwarzschild Strasse 2, 85748 Garching, Germany \\ 3 Dipartimento di Astronomia, Universitá di Trieste, via G.B. Tiepolo 11, 34131 Trieste, Italy \\ ${ }^{4}$ Observatoire de Paris-Meudon, GEPI, 92195 Meudon Cedex, France \\ 5 INAF Osservatorio Astronomico di Trieste, via G.B. Tiepolo 11, 34131 Trieste, Italy
}

Received 1 August 2003 / Accepted 12 March 2004

\begin{abstract}
We computed the evolution of the abundances of $\mathrm{O}, \mathrm{Mg}, \mathrm{Si}, \mathrm{Ca}, \mathrm{K}, \mathrm{Ti}, \mathrm{Sc}, \mathrm{Ni}, \mathrm{Mn}, \mathrm{Co}, \mathrm{Fe}$ and $\mathrm{Zn}$ in the Milky Way. We made use of the most widely adopted nucleosynthesis calculations and compared the model results with observational data with the aim of imposing constraints upon stellar yields. To best fit the data in the solar neighborhood, when adopting the Woosley \& Weaver (1995, ApJS, 101, 181) yields for massive stars and the Iwamoto et al. (1999, ApJS, 125, 439) ones for type Ia $\mathrm{SNe}$, it is required that: i) the $\mathrm{Mg}$ yields should be increased in stars with masses from 11 to $20 M_{\odot}$ and decreased in masses larger than $20 M_{\odot}$. The Mg yield should be also increased in SNe Ia. ii) The Si yields should be slightly increased in stars above $40 M_{\odot}$, whereas those of Ti should be increased between 11 and $20 M_{\odot}$ and above $30 M_{\odot}$. iii) The $\mathrm{Cr}$ and $\mathrm{Mn}$ yields should be increased in stars with masses in the range 11-20 $M_{\odot}$; iv) the Co yields in SNe Ia should be larger and smaller in stars in the range 11-20 $M_{\odot}$; v) the Ni yield from type Ia SNe should be decreased; vi) the Zn yield from type Ia SNe should be increased. vii) The yields of $\mathrm{O}$ (metallicity dependent $\mathrm{SN}$ models), $\mathrm{Ca}, \mathrm{Fe}, \mathrm{Ni}$, and $\mathrm{Zn}$ (the solar abundance case) in massive stars from Woosley \& Weaver (1995) are the best to fit the abundance patterns of these elements since they do not need any changes. We also adopted the yields by Nomoto et al. (1997, Nucl. Phys. A, 621, 467) and Limongi \& Chieffi (2003, ApJ, 592, 404) for massive stars and discuss the corrections required in these yields in order to fit the observations. Finally, the small spread in the $[\mathrm{el} / \mathrm{Fe}]$ ratios in the metallicity range from $[\mathrm{Fe} / \mathrm{H}]=-4.0$ up to -3.0 dex (Cayrel et al. 2004, A\&A, 416, 1117) is a clear sign that the halo of the Milky Way was well mixed even in the earliest phases of its evolution.
\end{abstract}

Key words. stars: abundances - galaxy: evolution

\section{Introduction}

Abundances and abundance ratios in very metal poor stars $([\mathrm{Fe} / \mathrm{H}]<-3.0 \mathrm{dex})$ are fundamental tools to understand the earliest phases of the evolution of the Milky Way as well as high redshift objects. Moreover, from the study of the $[\mathrm{el} / \mathrm{Fe}]$ versus $[\mathrm{Fe} / \mathrm{H}]$ relations one can infer very important constraints on stellar nucleosynthesis calculations, stellar lifetimes and the star formation history in galaxies (see Matteucci 2001). In particular, the study of very metal poor stars in the Galaxy allows us to understand the nucleosynthesis in massive stars and thus impose constraints on stellar models. Very few calculations are available for stars in the range 30-100 $M_{\odot}$ and one has to extrapolate for the yields in this mass range when calculating the chemical evolution of galaxies. For this reason it is of paramount importance to derive accurate abundances and abundance ratios in the extremely metal poor stars and this has become possible only recently by means of high

Send offprint requests to: P. François, e-mail: patrick.Francois@obspm.fr resolution spectrographs such as UVES installed at the VLT. Cayrel et al. (2004) have derived the abundances of several $\alpha$ and $\mathrm{Fe}$-peak elements for a sample of very metal poor giants ( $[\mathrm{Fe} / \mathrm{H}]$ from -4.0 to $-3.0 \mathrm{dex})$ thus allowing us to test chemical evolution models in this metallicity range never reached before. Several models of chemical evolution of the Milky Way are now available in the literature (Chiappini et al. 1997; Portinari \& Chiosi 1999; Goswami \& Prantzos 2000; Chang et al. 1999; Alibès et al. 2001) and they all share some important features such as the relaxation of the instantaneous recycling approximation (IRA), which is fundamental to follow the evolution of elements produced on long timescales, and the inclusion of type Ia SNe following the original prescriptions of Greggio \& Renzini (1983) and Matteucci \& Greggio (1986). These models can reproduce the majority of the properties of the solar neighborhood and the whole disk, in particular they all agree that the disk of the Milky Way should have formed inside-out and that the local disk assembled on a timescale of the order of 6-7 Gyr. These models also reproduce the abundance gradients observed along the Galactic disk 
as well as the distribution of gas and star formation. In this paper we plan to adopt an updated version of the two-infall model of Chiappini et al. (1997) and include several different stellar yields published so far to reproduce the most recent data on metal poor stars as well as the data for all metallicities. We will adopt a selected and accurate data sample. From the comparison between theory and observations we derive strong constraints upon the stellar yields which are still affected by large uncertainties. Especially uncertain are the yields of Fe-peak elements owing to the uncertain mass-cut, explosion energies, neutron fluxes mixing and possible fall-back of the expelled material. The comparison between the new data at very low metallicity and the detailed chemical evolution models will allow us to suggest how to correct the yields and gain information on the nucleosynthesis of high mass stars. A similar approach was presented in Argast et al. (2002) where the authors tried to constrain the yields of $\mathrm{O}, \mathrm{Mg}$ and Fe by fitting observations. However, they focused on a possible scatter present in the abundance ratios for $[\mathrm{Fe} / \mathrm{H}]<-2.5$. The new data for very metal poor stars imply only a small scatter, thus suggesting that the halo was indeed well mixed and allowing one to impose constraints on the nucleosynthesis of massive stars up to 30$35 M_{\odot}$ and thus extend the work of Argast et al. (2002), who could impose constraints only on the range $10-20 M_{\odot}$ owing to the lack of data at very low metallicities. However, by means of chemical evolution models one is able to impose constraints on the whole range of stellar masses up to $100 M_{\odot}$ since the most massive stars contribute to the solar chemical abundances even if we cannot impose precise constraints on their production ratios. To impose such constraints we would need to have measured abundance ratios for $[\mathrm{Fe} / \mathrm{H}]<-4.0 \mathrm{dex}$ (Audouze \& Silk 1995; Ryan 1996). The paper is organized as follows: in Sect. 2 we describe the data sample, in Sect. 3 the adopted chemical evolution model, in Sect. 4 the nucleosynthesis prescriptions. In Sect. 5 we show the comparison between models and observations and, in Sect. 6, some conclusions are drawn.

\section{Observational data}

We have adopted a data sample for stars in the solar neighborhood spanning a metallicity range from -4 to solar. In particular, for the very metal poor stars $([\mathrm{Fe} / \mathrm{H}]$ between -4 and -3$)$, we have considered the very recent results from the UVES Large Program "First Stars" (Cayrel et al. 2004). This sample is made up of 35 extremely metal-poor giants selected in the HK survey (Beers et al. 1992, 1999). The analysis has been made in a systematic and homogeneous way, from very high quality data, so that reliable trends of the abundances may be derived. It is important to note that the Beers et al. data (1992) is based on a very large survey $\left(1940 \mathrm{deg}^{2}\right)$. The detection of very metal poor stars is based on the strength of the $\mathrm{H}$ and $\mathrm{K}$ lines. The halo stars are rather uniformely distributed over the sky. The selection of the sample of Cayrel's stars is based on the estimation of their metallicity and their magnitude. The kinematics of these stars is such that they were born at very different places in the galactic halo. So, there is no possibility for a selection bias as could be found for a sample of bright disk dwarf stars located in the solar vicinity for which similar birthplaces and kinematics may be found.

Norris et al. (2001) introduced a factor $F$ (defined as $R \times(S / N) / \lambda$, where $R$ is the resolution of the spectrum and $S / N$ the signal to noise ratio), representative of the quality of the observational data. They estimated that $F$ values larger than 500 are now required to make progress in the understanding of the chemical history of our Galaxy. The data used in this study have $\mathrm{F}$ values ranging between 850 and 3250, which are larger than the values from other studies. These observations provide us with abundance ratios of unprecedented accuracy in this metallicity range.

For the abundances in the remaining range of $[\mathrm{Fe} / \mathrm{H}]$, we adopted already published data in the literature from various sources: Stephens (1999), Carney et al. (1997), Nissen \& Schuster (1997), Fulbright (2000), Gilroy et al. (1998), Gratton \& Sneden (1988, 1994), Ryan et al. (1991), Carretta et al. (2002), Edvardsson et al. (1993), McWilliam et al. (1995), Nissen et al. (2002), Matteucci et al. (1993, and references therein). All of these data are relative to the solar abundances of Grevesse \& Sauval (1998) with the exception of oxygen for which we adopted the new value of Allende Prieto et al. (2002).

\section{The chemical evolution model for the Milky Way}

The model for the Galaxy assumes two main infall episodes for the formation of the halo-thick disk, and the thin-disk, respectively. The timescale for the formation of the thin disk is much longer than that of the halo, implying that the infalling gas forming the thin disk comes not only from the halo but rather mainly from the intergalactic medium (e.g. Chiappini et al. 1997). The timescale for the formation of the thin disk is assumed to be a function of the galactocentric distance, leading to an inside-out picture for the Galaxy disk build-up, according to the original suggestion of Matteucci \& François (1989). The two-infall model differs from other models in the literature in two aspects: it considers an almost independent evolution between the halo and thin disk components (see also Pagel \& Tautvaisiene 1995), and it assumes a threshold in the star formation process (see Kennicutt 1989, 1998; Martin \& Kennicutt 2001). The model well reproduces the majority of observational constraints about the abundances of heavy elements both locally and in the whole disk.

If $G_{i}$ is the mass fraction of gas in the form of an element $i$, we can write the main equations of the model as:

$$
\begin{aligned}
& \dot{G}_{i}(t)=-\psi(r, t) X_{i}(r, t)+\int_{M_{\mathrm{L}}}^{M_{\mathrm{B} m}} \psi\left(t-\tau_{m}\right) Q_{m i}\left(t-\tau_{m}\right) \phi(m) \mathrm{d} m \\
& +A \int_{M_{\mathrm{B} m}}^{M_{\mathrm{B} M}} \phi\left(M_{\mathrm{B}}\right)\left[\int_{\mu_{\min }}^{0.5} f(\mu) \psi\left(t-\tau_{m 2}\right) Q_{m i}\left(t-\tau_{m 2}\right) \mathrm{d} \mu\right] \mathrm{d} M_{\mathrm{B}} \\
& \quad+(1-A) \int_{M_{\mathrm{B} m}}^{M_{\mathrm{B} M}} \psi\left(t-\tau_{m}\right) Q_{m i}\left(t-\tau_{m}\right) \phi(m) \mathrm{d} m \\
& \quad+\int_{M_{\mathrm{B} M}}^{M_{U}} \psi\left(t-\tau_{m}\right) Q_{m i}\left(t-\tau_{m}\right) \phi(m) \mathrm{d} m+X_{A_{i}} A(r, t)
\end{aligned}
$$

The star formation rate (SFR) adopted here is:

$\psi(r, t)=v(t)\left(\frac{\Sigma(r, t)}{\Sigma\left(r_{\odot}, t\right)}\right)^{2(k-1)}\left(\frac{\Sigma\left(r, t_{\mathrm{Gal}}\right)}{\Sigma(r, t)}\right)^{k-1} G_{\mathrm{gas}}^{k}(r, t)$ 
where $v(t)$ is the efficiency of the star formation process. $\Sigma(r, t)$ is the total surface mass density at a given radius $r$ and given time $t, \Sigma\left(r_{\odot}, t\right)$ is the total surface mass density at the solar position, $G_{\text {gas }}(r, t)$ is the surface gas density normalized to the present time total surface mass density in the disk $\Sigma_{\mathrm{D}}\left(r, t_{\mathrm{Gal}}\right)$, $t_{\mathrm{Gal}}=13 \mathrm{Gyr}$ is the age of the Galaxy, $r_{\odot}=8 \mathrm{kpc}$ is the assumed solar galactocentric distance (see Reid 1993).

The gas surface density exponent, $k$, is set equal to 1.5 , to ensure a good fit to the observational constraints in the solar vicinity. This value is also in agreement with the observational results of Kennicutt (1998), and with $N$-body simulation results by Gerritsen \& Icke (1997). The star formation efficiency is set to $v=2 \mathrm{Gyr}^{-1}$, for the Galactic halo, whereas it is $v=1 \mathrm{Gyr}^{-1}$ for the disk; this is to ensure the best fit to the observational features in the solar vicinity. The star formation rate becomes zero when the gas surface density drops below a certain critical threshold (see Chiappini et al. 2001 for details). The assumption of such a threshold density naturally produces an hiatus in the SFR between the halo-thick disk phase and the thin disk phase. This discontinuity in the SFR seems to be observed in the $[\mathrm{Fe} / \mathrm{O}]$ vs. $[\mathrm{O} / \mathrm{H}]$ (Gratton et al. 2000) and in the $[\mathrm{Fe} / \mathrm{Mg}]$ vs. $[\mathrm{Mg} / \mathrm{H}]$ (Fuhrmann 1998) plots. The initial mass function (IMF) is that of Scalo (1986) and is assumed to be constant in time and space.

The SNeIa rate has been computed following Greggio \& Renzini (1983) and Matteucci \& Greggio (1986) and is expressed as:

$R_{\mathrm{SNeIa}}=A \int_{M_{\mathrm{B}_{m}}}^{M_{\mathrm{B}_{M}}} \phi\left(M_{\mathrm{B}}\right) \int_{\mu_{m}}^{0.5} f(\mu) \psi\left(t-\tau_{M_{2}}\right) \mathrm{d} \mu \mathrm{d} M_{\mathrm{B}}$,

where $M_{2}$ is the mass of the secondary, $M_{\mathrm{B}}$ is the total mass of the binary system, $\mu=M_{2} / M_{\mathrm{B}}, \mu_{m}=\max \left\{M_{2}(t) / M_{\mathrm{B}},\left(M_{\mathrm{B}}-\right.\right.$ $\left.\left.0.5 M_{\mathrm{B}_{M}}\right) / M_{\mathrm{B}}\right\}, M_{\mathrm{B}_{m}}=3 M_{\odot}, M_{\mathrm{B}_{M}}=16 M_{\odot}$. The IMF is represented by $\phi\left(M_{\mathrm{B}}\right)$ and refers to the total mass of the binary system for the computation of the SNIa rate, $f(\mu)$ is the distribution function for the mass fraction of the secondary, $f(\mu)=2^{1+\gamma}(1+\gamma) \mu^{\gamma}$, with $\gamma=2 ; A=0.05$ is the fraction of systems with total mass in the appropriate range, which give rise to SNIa events. This quantity is fixed by reproducing the observed SNe Ia rate at the present epoch (Cappellaro et al. 1999; see also Madau et al. 1998).

The term $A(r, t)$ represents the accretion term and is defined as:

$A(r, t)=a(r) \mathrm{e}^{-t / \tau_{\mathrm{H}}(r)}+b(r) \mathrm{e}^{-\left(t-t_{\max }\right) / \tau_{\mathrm{D}}(r)}$

$X_{A_{i}}$ are the abundances in the infalling material, which is assumed to be primordial, while $t_{\max }=1 \mathrm{Gyr}$ is the time for the maximum infall on the thin disk, $\tau_{\mathrm{H}}=2.0 \mathrm{Gyrs}$ is the time scale for the formation of the halo thick-disk and $\tau(r)$ is the timescale for the formation of the thin disk and is a function of the galactocentric distance (formation inside-out, Matteucci \& François 1989; Chiappini et al. 2001). In particular, we assume that:

$\tau_{\mathrm{D}}=1.033 r(\mathrm{kpc})-1.267 \mathrm{Gyr}$.

Finally, the coefficients $a(r)$ and $b(r)$ are obtained by imposing a fit to the current total surface mass density as a function of galactocentric distance. In particular, $b(r)$ is assumed to be different from zero only for $t \geq t_{\max }$.

\section{Nucleosynthesis prescriptions}

We divide stars into three fundamental mass ranges: i) very low mass stars $\left(M<0.8 M_{\odot}\right)$; ii) low and intermediate mass stars $\left(0.8 \leq M / M_{\odot} \leq 8\right)$ and iii) massive stars $\left(M>8 M_{\odot}\right)$. Very low mass stars do not contribute to the chemical enrichment but only to lock up gas. Low and intermediate mass stars contribute to $\mathrm{He},{ }^{12} \mathrm{C},{ }^{13} \mathrm{C},{ }^{14} \mathrm{~N}$ and to some s-process elements (see Travaglio et al. 1999). Massive stars are responsible for the formation of the bulk of $\alpha$-elements $(\mathrm{O}, \mathrm{Mg}, \mathrm{Ne}, \mathrm{Si}, \mathrm{S}$, $\mathrm{Ca}, \mathrm{Ti}$ ) plus some $\mathrm{Fe}$ and $\mathrm{Fe}$-peak elements whose yields are rather uncertain. These stars end their lives as type II supernovae. Type Ia SNe (C-O white dwarfs in binary systems) are instead considered to be responsible for the production of the bulk of $\mathrm{Fe}$ and Fe-peak elements.

In this paper we adopt the nucleosynthesis prescriptions of van den Hoek \& Groenewegen (1997) for single low and intermediate mass stars, of Iwamoto et al. (1999) for the yields from type Ia SNe (model W7) and the yields of Woosley \& Weaver (1995) (hereafter WW95, their case A for stars below $30 M_{\odot}$ and their case B for stars between 30 and $40 M_{\odot}$ ), Nomoto et al. (1997) (hereafter N97) and Limongi \& Chieffi (2003) (hereafter LC03) for massive stars. While WW95 have provided yields for different initial stellar metallicities, those of N97 and LC03 refer only to the solar chemical composition. Generally, yields of primary elements, namely those elements produced starting directly from the $\mathrm{H}$ and $\mathrm{He}$ through the chain of hydrostatic burnings in stars, depend only slightly on the initial stellar metallicity. Therefore, we have chosen to consider the WW95 yields of primary elements that refer to the solar chemical composition with the exception of oxygen (see next section). For $\mathrm{Zn}$, which has a more complex nucleosynthetic origin, being partly produced in explosive nucleosynthesis and partly being manufactured as an s-process element in massive stars, we adopted WW95 values for the explosive nucleosynthesis in massive stars and the prescriptions of Matteucci et al. (1993) for the other components (nucleosynthesis in type Ia $\mathrm{SNe}$ and quiescent He-burning in massive stars during which weak s-processing takes place). In particular, these authors suggested that the yield of $\mathrm{Zn}$ from type Ia SNe should be higher than predicted and that it represents the major component in the $\mathrm{Zn}$ production. This element is quite important since is the best tracer of metallicity in high redshift objects such as Damped Lyman- $\alpha$ systems (DLA) and Lyman-break galaxies, owing to the fact that it is only very slightly depleted into dust. Stellar yields, especially those of Fe-peak elements, are still quite uncertain since they strongly depend upon the choice of the mass cut between ejecta and the proto-neutron stars, the explosion energies and the neutron flux mixing. On the other hand, the yields of elements produced during hydrostatic burnings such as $\mathrm{O}$ and $\mathrm{Mg}$ should be better known. However, the $\mathrm{Mg}$ yield is more sensitive than $\mathrm{O}$ to the treatment of convection used by the different authors. In this paper, we have adopted a stellar mass range of $0.1-100 M_{\odot}$ and since the available nucleosynthesis prescriptions go only until $70 M_{\odot}$ (N97) we have kept 

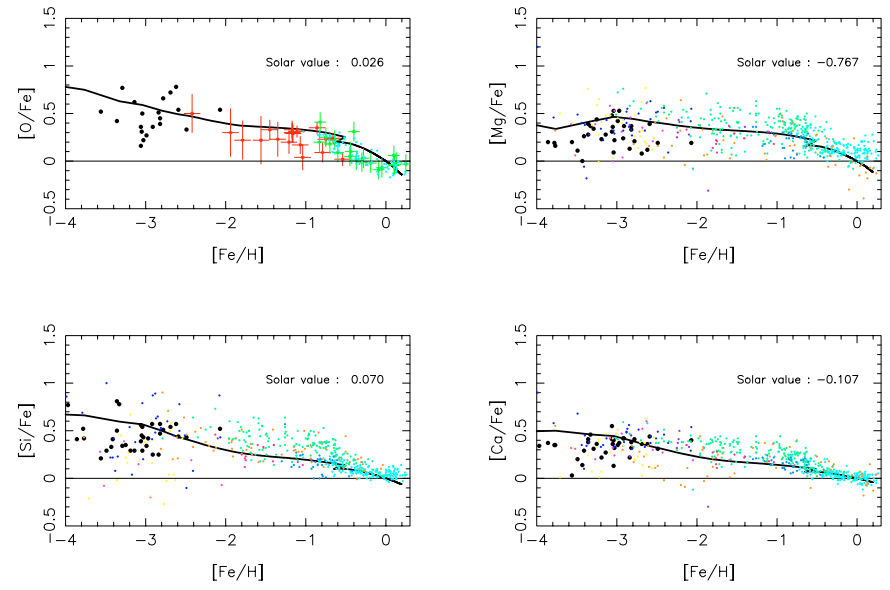

Fig. 1. $[\mathrm{el} / \mathrm{Fe}]$ versus $[\mathrm{Fe} / \mathrm{H}]$ for several $\alpha$ - elements compared to a large compilation of data (see text). The black dots represent the new data of Cayrel et al. (2004). Here we have adopted the yields of WW95 for massive stars and $\mathrm{N} 97$ for type Ia SNe taken as per their models.The model predictions are normalized to the predicted solar abundances, namely those predicted at $4.5 \mathrm{Gyr}$ ago. In a corner of each panel we show our predicted solar abundance ratios. In some cases we fit the behavior of $[\mathrm{el} / \mathrm{Fe}]$ vs. $[\mathrm{Fe} / \mathrm{H}]$ but not the solar value, as it is the case for $\mathrm{Mg}$.
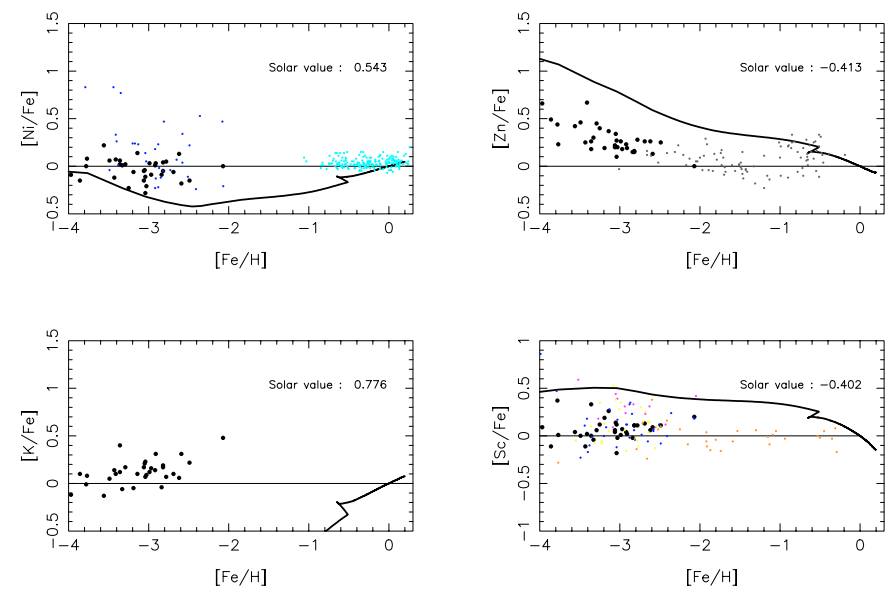

Fig. 2. The same as in Fig. 1 for several Fe- peak elements. The yields are taken at as per their models.

the yields constant and equal to the value corresponding to the largest computed stellar mass for which the yields are available.

\section{Model results}

In Figs. 1-3 we show the model predictions for several elements (O, Mg, Si, Ca, K, Ti, Ni, Sc, Cr, Mn, Co and Zn), in particular for the relations $[\mathrm{el} / \mathrm{Fe}]$ versus $[\mathrm{Fe} / \mathrm{H}]$ compared with the observational data described before. The yields that we have adopted are those of the SNII of WW95 with initial solar chemical composition, with the exception of oxygen.

For this element we adopted the metallicity-dependent yields of WW95 since they best fit the new data of oxygen at very low metallicity. Goswami \& Prantzos (2000) had already published the predictions for $[\mathrm{O} / \mathrm{Fe}]$ vs. $[\mathrm{Fe} / \mathrm{H}]$ obtained by
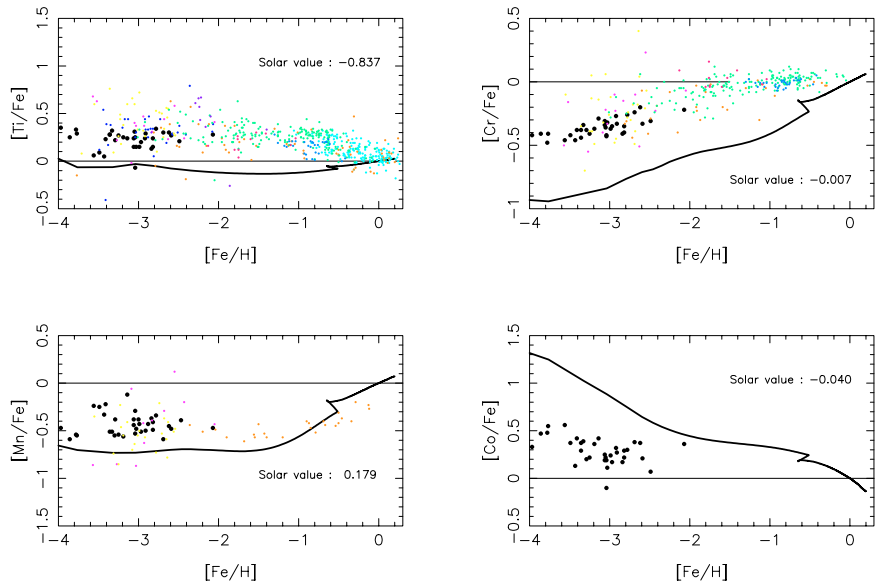

Fig. 3. The same as in Fig. 1 for several Fe- peak elements. The yields are taken as per their models.

using both the oxygen yields of WW95 as a function of metallicity as well as those for the solar chemical composition, and although the differences between these two cases are generally small, especially for primary elements, it was evident from their figures that the metallicity-dependent case was the best to reproduce the oxygen data available then. For type Ia SNe we took the yields of Iwamoto et al. (1999) from their model W7. In all our models we have normalized the predicted abundances to the predicted solar abundances, which reflect the abundances in the gas $4.5 \mathrm{Gyr}$ ago. Therefore, the predicted curve includes the 0,0 point. In each panel of Figs. $1-3$ we print in the top right the solar $[\mathrm{el} / \mathrm{Fe}]$ ratio that we predict for the time of formation of the solar system and relative to the observed solar abundances (Grevesse \& Sauval 1998 for all the elements except oxygen for which we adopt the newer estimates of Holweger 2001 and Allende-Prieto et al. 2001). A good model of chemical evolution of the Milky Way should be able to reproduce both the abundance patterns and the absolute solar abundances. As one can see from Figs. 1-3 the $\mathrm{O}, \mathrm{Mg}, \mathrm{Si}$ and $\mathrm{Ca}$ behaviours are very well fitted as are their solar values with the exception of the $[\mathrm{Mg} / \mathrm{Fe}]$ ratio, which is largely underestimated, owing to the too low $\mathrm{Mg}$ yields predicted for massive stars, a problem common to all chemical evolution models (e.g. Chiappini et al. 1999; Thomas et al. 1999). On the other hand, for the other elements ( $\mathrm{Ni}, \mathrm{Zn}, \mathrm{K}, \mathrm{Sc}, \mathrm{Ti}, \mathrm{Cr}, \mathrm{Mn}$ and $\mathrm{Co}$ ) the trends are not well reproduced and, with the exception of $\mathrm{Co}, \mathrm{Mn}$ and $\mathrm{Cr}$, not even the predicted solar abundance ratios are confirmed. Therefore, there is a clear indication that it is necessary to modify the yields, especially those of the Fe-peak elements. In Figs. 4-6 we show the predictions obtained with the yields modified "ad hoc" to fit the data, according to the prescriptions given in Fig. 7, where we show the ratios between the suggested and the published yields of WW95 for SNII as well as those of Iwamoto et al. (1999), model W7, for the type Ia SNe.The suggested yields are shown in Tables 1 and 2 .

As an example we examine the treatment adopted for the yields of $\mathrm{K}$ which was applied to fit the variation of [K/Fe] vs. $[\mathrm{Fe} / \mathrm{H}]$ found in the halo stars. Figures 2, 5 and 7 for $\mathrm{K}$ shows what treatment has been done to fit the data points. Using the yields for SNII (WW95) and SNIa (Nomoto) gives a too high 

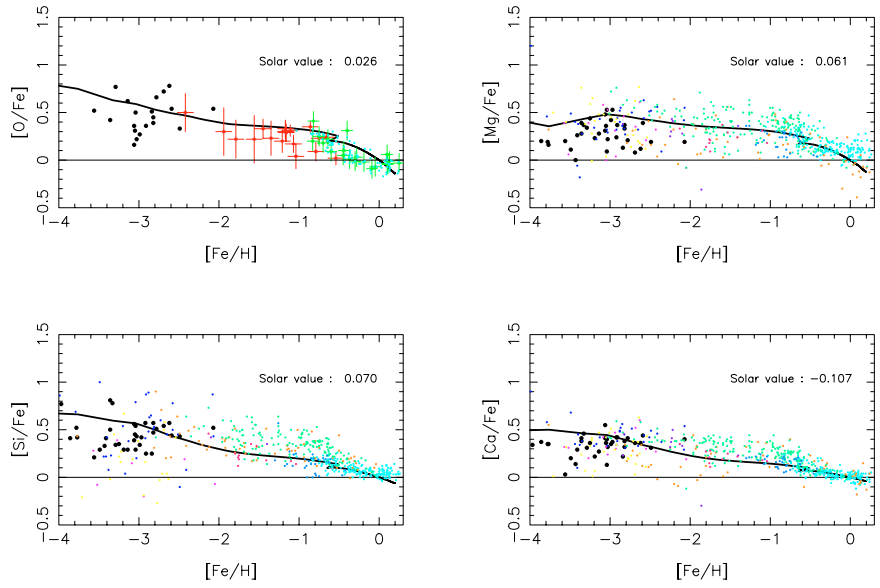

Fig. 4. $[\mathrm{el} / \mathrm{Fe}]$ versus $[\mathrm{Fe} / \mathrm{H}]$ for several $\alpha$-elements, as predicted by adopting the corrected yields, compared with a large data sample (Table 1). The model predictions are normalized to the predicted solar abundances, namely those predicted for the gas at $4.5 \mathrm{Gyr}$ ago. As one can see, the model reproduces now both the behavior of abundance ratios and the solar abundances.
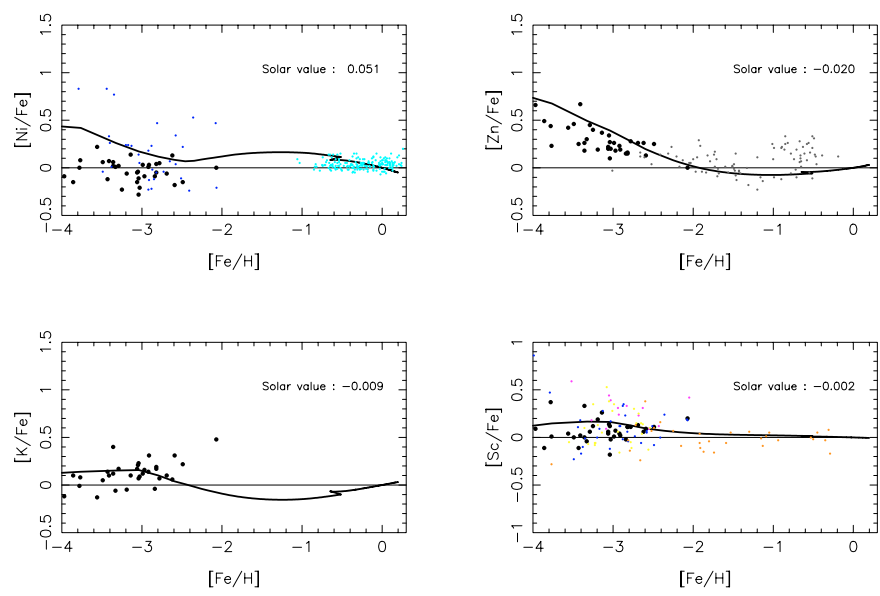

Fig. 5. The same as Fig. 2 for some Fe-peak elements.

value of $[\mathrm{K} / \mathrm{Fe}]$ at the solar birth $([\mathrm{K} / \mathrm{Fe}]=0.776)$. In Fig. 2, an increasing slope from metal-poor stars to solar metallicity reveals that the production ratio SNII/SNIa is much too high (we have a rather similar case for $\mathrm{Cr}, \mathrm{Mn}$ and $\mathrm{Ni}$ ). Therefore, a decrease of this ratio by a factor of 8 allows us to get a decreasing $[\mathrm{K} / \mathrm{Fe}]$ as a function of increasing metallicity. The final adjustment for the SNII yields is used to get a $\operatorname{good}[\mathrm{Fe} / \mathrm{H}]$ value at solar birth. As $[\mathrm{K} / \mathrm{Fe}]$ is constant in the metal-poor stars, there is no need to change the SNII yields as a function of mass.

We have performed a series of tests to evaluate the sensitivity of the yields found in Tables 1 and 2 to different assumptions of the models of chemical evolution of the Galaxy. First, we changed the efficiency of the Star Formation Rate by a factor of 2. It changes the absolute abundances and then affects the Fe abundance found at solar birth. The impact on the abundance ratios is less than $0.03 \mathrm{dex}$. We also estimated the impact of the change of the IMF Scalo coefficient by \pm 0.2 and found that the impact on the abundance ratio is less than 0.10 dex. We performed a test on the relative sensitivity of the yields of the
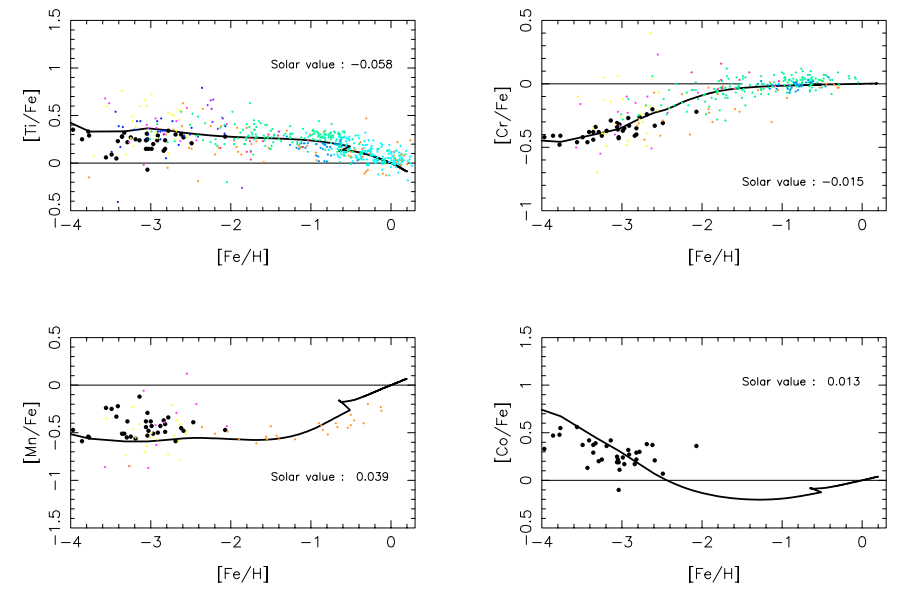

Fig. 6. The same as Fig. 2 for some Fe-peak elements.

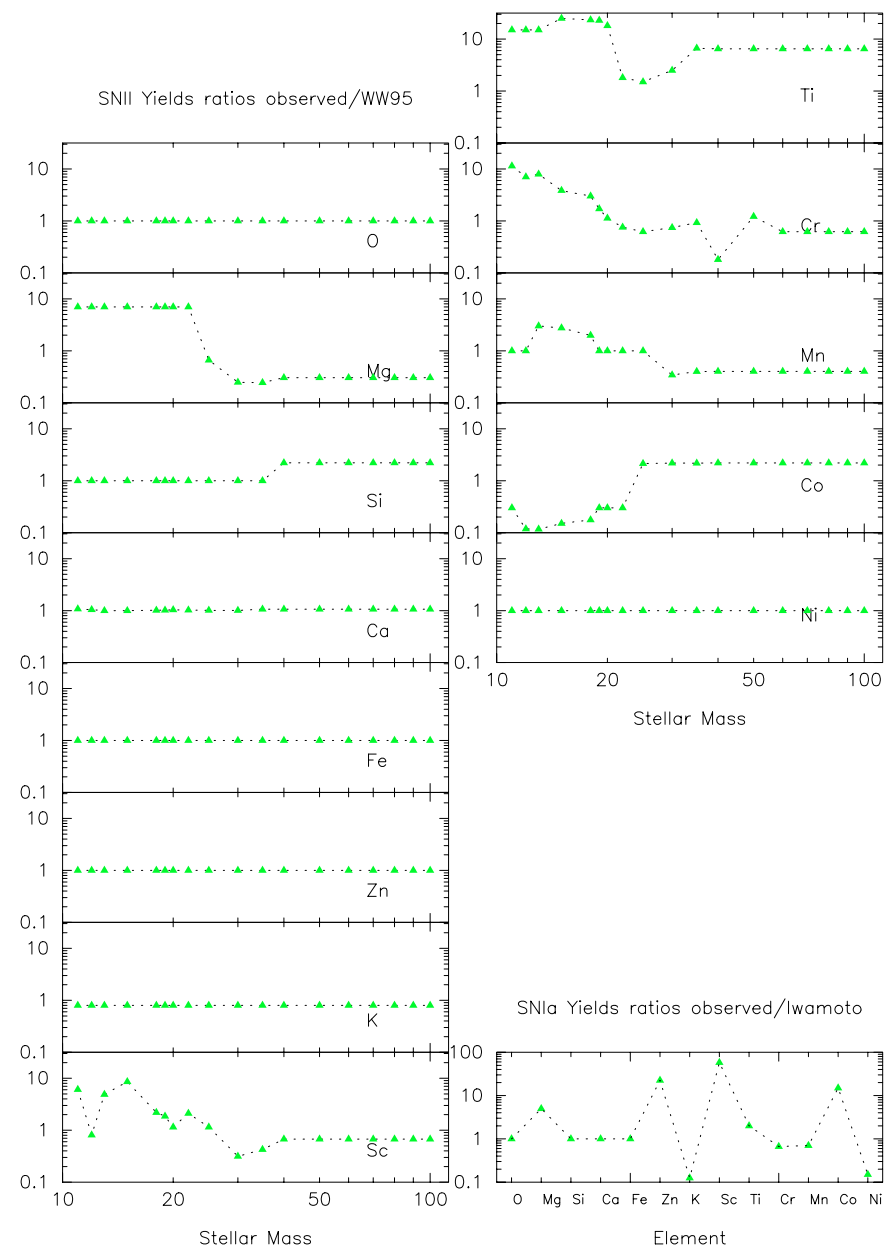

Fig. 7. Ratios between the yields we adopted to obtain the best fit to the data and the yields of WW95 for massive stars and those of Iwamoto et al. (1999) for the nucleosynthesis in type Ia SNe. Notice that the oxygen yields are those of WW95 as a function of metallicity whereas all the other yields of WW95 refer to the solar chemical composition.

elements for different mass ranges. We divided the masses in 3 mass ranges. For each element and each mass range we multiplied or divided the yields of Tables 1 and 2 iteratively and then determined a factor such that the yields of an element in a given mass range can be multiplied or divided by this factor 
Table 1. The stellar yields (expressed in solar masses) as derived in this paper, namely those that produce the best agreement with observations. Yields for $\mathrm{O}$ are identical to metal dependent yields of WW95.

\begin{tabular}{cccccc}
\hline \hline$m\left(M_{\odot}\right)$ & $\mathrm{Mg}$ & $\mathrm{Si}$ & $\mathrm{Ca}$ & $\mathrm{Fe}$ & $\mathrm{Zn}$ \\
\hline 11.00 & $.6440 \mathrm{E}-01$ & $.2170 \mathrm{E}-01$ & $.1400 \mathrm{E}-02$ & $.8000 \mathrm{E}-01$ & $.3510 \mathrm{E}-04$ \\
12.00 & $.5740 \mathrm{E}-01$ & $.9090 \mathrm{E}-01$ & $.1500 \mathrm{E}-01$ & $.5500 \mathrm{E}-01$ & $.3360 \mathrm{E}-04$ \\
13.00 & $.1148 \mathrm{E}+00$ & $.5850 \mathrm{E}-01$ & $.3600 \mathrm{E}-02$ & $.1460 \mathrm{E}+00$ & $.4500 \mathrm{E}-04$ \\
15.00 & $.1869 \mathrm{E}+00$ & $.1100 \mathrm{E}+00$ & $.1110 \mathrm{E}-01$ & $.1297 \mathrm{E}+00$ & $.7280 \mathrm{E}-04$ \\
18.00 & $.3864 \mathrm{E}+00$ & $.1370 \mathrm{E}+00$ & $.6900 \mathrm{E}-02$ & $.8300 \mathrm{E}-01$ & $.1060 \mathrm{E}-03$ \\
19.00 & $.1757 \mathrm{E}+00$ & $.2770 \mathrm{E}+00$ & $.1380 \mathrm{E}-01$ & $.1170 \mathrm{E}+00$ & $.1560 \mathrm{E}-03$ \\
20.00 & $.2191 \mathrm{E}+00$ & $.2880 \mathrm{E}+00$ & $.1480 \mathrm{E}-01$ & $.1060 \mathrm{E}+00$ & $.3160 \mathrm{E}-03$ \\
22.00 & $.2912 \mathrm{E}+00$ & $.3560 \mathrm{E}+00$ & $.1770 \mathrm{E}-01$ & $.2225 \mathrm{E}+00$ & $.3240 \mathrm{E}-03$ \\
25.00 & $.7000 \mathrm{E}-01$ & $.3150 \mathrm{E}+00$ & $.1690 \mathrm{E}-01$ & $.1500 \mathrm{E}+00$ & $.4070 \mathrm{E}-03$ \\
30.00 & $.7000 \mathrm{E}-01$ & $.3160 \mathrm{E}+00$ & $.1340 \mathrm{E}-01$ & $.2500 \mathrm{E}-01$ & $.3818 \mathrm{E}-03$ \\
35.00 & $.7000 \mathrm{E}-01$ & $.1120 \mathrm{E}+00$ & $.1600 \mathrm{E}-02$ & $.2770 \mathrm{E}-01$ & $.4176 \mathrm{E}-03$ \\
40.00 & $.7000 \mathrm{E}-01$ & $.5460 \mathrm{E}-01$ & $.1600 \mathrm{E}-02$ & $.2830 \mathrm{E}-01$ & $.3710 \mathrm{E}-03$ \\
50.00 & $.7000 \mathrm{E}-01$ & $.5460 \mathrm{E}-01$ & $.1600 \mathrm{E}-02$ & $.2830 \mathrm{E}-01$ & $.3710 \mathrm{E}-03$ \\
60.00 & $.7000 \mathrm{E}-01$ & $.5460 \mathrm{E}-01$ & $.1600 \mathrm{E}-02$ & $.2830 \mathrm{E}-01$ & $.3710 \mathrm{E}-03$ \\
70.00 & $.7000 \mathrm{E}-01$ & $.5460 \mathrm{E}-01$ & $.1600 \mathrm{E}-02$ & $.2830 \mathrm{E}-01$ & $.3710 \mathrm{E}-03$ \\
80.00 & $.7000 \mathrm{E}-01$ & $.5460 \mathrm{E}-01$ & $.1600 \mathrm{E}-02$ & $.2830 \mathrm{E}-01$ & $.3710 \mathrm{E}-03$ \\
90.00 & $.7000 \mathrm{E}-01$ & $.5460 \mathrm{E}-01$ & $.1600 \mathrm{E}-02$ & $.2830 \mathrm{E}-01$ & $.3710 \mathrm{E}-03$ \\
100.00 & $.7000 \mathrm{E}-01$ & $.5460 \mathrm{E}-01$ & $.1600 \mathrm{E}-02$ & $.2830 \mathrm{E}-01$ & $.3710 \mathrm{E}-03$ \\
\hline
\end{tabular}

Table 2. The stellar yields (expressed in solar masses) as derived in this paper, namely those that produce the best agreement with observations.

\begin{tabular}{cccccccc}
\hline \hline$m\left(M_{\odot}\right)$ & $\mathrm{K}$ & $\mathrm{Sc}$ & $\mathrm{Ti}$ & $\mathrm{Cr}$ & $\mathrm{Mn}$ & $\mathrm{Co}$ & $\mathrm{Ni}$ \\
\hline 11.00 & $.4040 \mathrm{E}-04$ & $.2564 \mathrm{E}-05$ & $.3300 \mathrm{E}-03$ & $.2037 \mathrm{E}-02$ & $.1290 \mathrm{E}-03$ & $.1272 \mathrm{E}-03$ & $.1670 \mathrm{E}-01$ \\
12.00 & $.1712 \mathrm{E}-02$ & $.2714 \mathrm{E}-05$ & $.3960 \mathrm{E}-03$ & $.2091 \mathrm{E}-02$ & $.1400 \mathrm{E}-03$ & $.2010 \mathrm{E}-04$ & $.7000 \mathrm{E}-02$ \\
13.00 & $.6160 \mathrm{E}-04$ & $.2553 \mathrm{E}-05$ & $.3990 \mathrm{E}-03$ & $.1929 \mathrm{E}-02$ & $.4490 \mathrm{E}-03$ & $.2019 \mathrm{E}-04$ & $.1120 \mathrm{E}-01$ \\
15.00 & $.2544 \mathrm{E}-03$ & $.6751 \mathrm{E}-05$ & $.7515 \mathrm{E}-03$ & $.1371 \mathrm{E}-02$ & $.4710 \mathrm{E}-03$ & $.3030 \mathrm{E}-04$ & $.6800 \mathrm{E}-02$ \\
18.00 & $.1176 \mathrm{E}-03$ & $.2415 \mathrm{E}-05$ & $.8400 \mathrm{E}-03$ & $.8910 \mathrm{E}-03$ & $.4040 \mathrm{E}-03$ & $.4290 \mathrm{E}-04$ & $.3760 \mathrm{E}-02$ \\
19.00 & $.6952 \mathrm{E}-03$ & $.6038 \mathrm{E}-05$ & $.8670 \mathrm{E}-03$ & $.7980 \mathrm{E}-03$ & $.2060 \mathrm{E}-03$ & $.7710 \mathrm{E}-04$ & $.4680 \mathrm{E}-02$ \\
20.00 & $.7496 \mathrm{E}-03$ & $.1086 \mathrm{E}-04$ & $.8700 \mathrm{E}-03$ & $.5400 \mathrm{E}-03$ & $.2130 \mathrm{E}-03$ & $.8160 \mathrm{E}-04$ & $.4440 \mathrm{E}-02$ \\
22.00 & $.1200 \mathrm{E}-02$ & $.2530 \mathrm{E}-04$ & $.8940 \mathrm{E}-04$ & $.4050 \mathrm{E}-03$ & $.2300 \mathrm{E}-03$ & $.8820 \mathrm{E}-04$ & $.1120 \mathrm{E}-01$ \\
25.00 & $.4032 \mathrm{E}-03$ & $.2622 \mathrm{E}-05$ & $.7500 \mathrm{E}-04$ & $.3900 \mathrm{E}-03$ & $.2580 \mathrm{E}-03$ & $.7500 \mathrm{E}-03$ & $.7590 \mathrm{E}-02$ \\
30.00 & $.2648 \mathrm{E}-03$ & $.1610 \mathrm{E}-05$ & $.1500 \mathrm{E}-03$ & $.4500 \mathrm{E}-03$ & $.1040 \mathrm{E}-03$ & $.8070 \mathrm{E}-03$ & $.3350 \mathrm{E}-01$ \\
35.00 & $.1328 \mathrm{E}-03$ & $.1345 \mathrm{E}-05$ & $.4500 \mathrm{E}-03$ & $.4500 \mathrm{E}-03$ & $.1330 \mathrm{E}-03$ & $.7950 \mathrm{E}-03$ & $.2040 \mathrm{E}-02$ \\
40.00 & $.8960 \mathrm{E}-04$ & $.1621 \mathrm{E}-05$ & $.4560 \mathrm{E}-03$ & $.9120 \mathrm{E}-04$ & $.1340 \mathrm{E}-03$ & $.8250 \mathrm{E}-03$ & $.2120 \mathrm{E}-02$ \\
50.00 & $.8960 \mathrm{E}-04$ & $.1621 \mathrm{E}-05$ & $.4560 \mathrm{E}-03$ & $.6120 \mathrm{E}-04$ & $.1340 \mathrm{E}-03$ & $.8250 \mathrm{E}-03$ & $.2120 \mathrm{E}-02$ \\
60.00 & $.8960 \mathrm{E}-04$ & $.1621 \mathrm{E}-05$ & $.4560 \mathrm{E}-03$ & $.3120 \mathrm{E}-04$ & $.1340 \mathrm{E}-03$ & $.8250 \mathrm{E}-03$ & $.2120 \mathrm{E}-02$ \\
70.00 & $.8960 \mathrm{E}-04$ & $.1621 \mathrm{E}-05$ & $.4560 \mathrm{E}-03$ & $.3120 \mathrm{E}-04$ & $.1340 \mathrm{E}-03$ & $.8250 \mathrm{E}-03$ & $.2120 \mathrm{E}-02$ \\
80.00 & $.8960 \mathrm{E}-04$ & $.1621 \mathrm{E}-05$ & $.4560 \mathrm{E}-03$ & $.3120 \mathrm{E}-04$ & $.1340 \mathrm{E}-03$ & $.8250 \mathrm{E}-03$ & $.2120 \mathrm{E}-02$ \\
90.00 & $.8960 \mathrm{E}-04$ & $.1621 \mathrm{E}-05$ & $.4560 \mathrm{E}-03$ & $.3120 \mathrm{E}-04$ & $.1340 \mathrm{E}-03$ & $.8250 \mathrm{E}-03$ & $.2120 \mathrm{E}-02$ \\
100.00 & $.8960 \mathrm{E}-04$ & $.1621 \mathrm{E}-05$ & $.4560 \mathrm{E}-03$ & $.3120 \mathrm{E}-04$ & $.1340 \mathrm{E}-03$ & $.8250 \mathrm{E}-03$ & $.2120 \mathrm{E}-02$ \\
\hline
\end{tabular}

without giving abundance ratios that are not in agreement with the observational data. These factors are given in Table 3 . Fe is not included in this table as it has been used as a reference element in computing the variations of the other yields.

As it is evident from Fig. 7, the yields that did not need any revision relative to the prescriptions of WW95 are those of $\mathrm{O}$ (computed as a function of metallicity), $\mathrm{Fe}, \mathrm{Ca}, \mathrm{Zn}$, $\mathrm{Ni}$ and $\mathrm{K}$ (corresponding to the solar chemical composition). For all other elements $(\mathrm{Mg}, \mathrm{Si}, \mathrm{K}, \mathrm{Ti}, \mathrm{Sc}, \mathrm{Cr}, \mathrm{Mn}, \mathrm{Co})$ some variations of the WW95 yields are required. In particular, the $\mathrm{Mg}$ yields predicted by the available nucleosynthesis calculations for massive stars need revision to reproduce the solar abundance of this element. Starting from the calculations of WW95, one needs to assume that the Mg yields from stars in the range $11-20 M_{\odot}$ should be roughly a factor of 7 higher than predicted whereas those from stars larger than $20 M_{\odot}$ should be lower than predicted (a factor of 2 on average). At the same time, to preserve the observed pattern of $[\mathrm{Mg} / \mathrm{Fe}]$ vs. $[\mathrm{Fe} / \mathrm{H}]$ one needs also to increase the $\mathrm{Mg}$ yields from type Ia $\mathrm{SNe}$ by a factor of 5 . The yields of $\mathrm{K}$ should be multiplied by a factor of 0.8 over the whole mass range in the case of WW95. As we can see in Figs. 8 and 9, where we show how one needs to modify the yields to reproduce the observations if one uses the yields of N97 and LC03, respectively, the same type of corrections should be applied to the other examined type II SN yields. We recall that these sets of yields (N97 and LC03) were computed only for solar chemical composition. Also in these cases, the $\mathrm{Mg}$ yields need to be increased below $20 M_{\odot}$, and decreased above $20 M_{\odot}$, although for the yields of LC03 the required increase is smaller than in WW95. Therefore, one can conclude 
Table 3. Sensitivity factors for the yields found in SNII: these factors give the amount by which the yields given in Tables 1 and 2 can be multiplied or divided such that the results of the model still give fair fits to the data.

\begin{tabular}{cccc}
\hline \hline Elt & 11 to $19 M_{\odot}$ & 20 to $30 M_{\odot}$ & 35 to $100 M_{\odot}$ \\
\hline $\mathrm{O}$ & 2 & 2 & 2 \\
$\mathrm{Mg}$ & 2 & 2 & 2 \\
$\mathrm{Si}$ & 3 & 3 & 3 \\
$\mathrm{Ca}$ & 2 & 2 & 5 \\
$\mathrm{Zn}$ & 5 & 2 & 2 \\
$\mathrm{~K}$ & 2 & 2 & 2 \\
$\mathrm{Sc}$ & 2 & 2 & 2 \\
$\mathrm{Ti}$ & 2 & 2 & 2 \\
$\mathrm{Cr}$ & 2 & 2 & 5 \\
$\mathrm{Mn}$ & 2 & 2 & 2 \\
$\mathrm{Co}$ & 2 & 5 & 2 \\
$\mathrm{Ni}$ & 2 & 2 & 2 \\
\hline
\end{tabular}

that the predicted $\mathrm{Mg}$ yields, either from type Ia or type II SNe below $20 M_{\odot}$, are too low. Another $\alpha$-element which seems largely underestimated over the whole mass range, in the three sets of yields (WW95, N97, LC03), is titanium, especially in the range $11-20 M_{\odot}$. Concerning $\mathrm{Si}$, only the yields of the very massive stars $\left(M>40 M_{\odot}\right)$ should be increased by a factor of 2, in the case of WW95 yields, whereas in the case of N97 they should be increased, especially in the range $15-25 M_{\odot}$ and lowered for more massive stars. The yields of Si LC03 should be only slightly increased in the range $20-30 M_{\odot}$ and decreased by a factor of $\sim 4$ for larger masses.

It is not easy to envisage how to obtain the requested yields from nucleosynthesis since the $\alpha$-elements $\mathrm{O}, \mathrm{Mg}, \mathrm{Si}$ and $\mathrm{Ca}$ are produced in different nuclear environments: $\mathrm{O}$ in He-burning, $\mathrm{Mg}$ in $\mathrm{C}$-burning and $\mathrm{Si}$ and $\mathrm{Ca}$ in explosive O-burning and in explosive incomplete Si-burning. In addition, the amount $\mathrm{Si}$ and $\mathrm{Ca}$ can depend on the choice of the mass cut whereas $\mathrm{O}$ and $\mathrm{Mg}$ do not. Certainly the rate of the ${ }^{12} \mathrm{C}(\alpha, \gamma){ }^{16} \mathrm{O}$ reaction is a very important parameter in determining not only the amounts of $\mathrm{O}$ and $\mathrm{Mg}$ but also the ratio between elements such as $\mathrm{Ne}, \mathrm{Na}, \mathrm{Mg}$ and $\mathrm{Al}$ relative to $\mathrm{Si}, \mathrm{S}$, Ar and Ca (Imbriani et al. 2001). A recent paper by Rauscher et al. (2002) adopting up to date experimental and theoretical nuclear data, new opacity tables and updated nuclear reaction network and considering stellar evolution with mass loss provided $\mathrm{Mg}$ yields higher than the previous ones of WW95 but still not high enough to reproduce the $\mathrm{Mg}$ data (they should still be increased by a factor of 3). On the other hand, their yields for a $25 M_{\odot}$ supernova are too high by a factor of 2 . The yields of $\mathrm{Cr}$ and $\mathrm{Mn}$ should be higher in the mass range 13-30 $M_{\odot}$ and slightly lower for more massive stars than predicted by WW95. Finally, the yields of Co of WW95 should be lowered in the range 11-22 $M_{\odot}$ and increased for more massive stars, and the yield of Sc should be generally increased in the same mass range and decreased for larger masses. The yields of Fe from WW95 relative to the case of solar chemical composition are very good, whereas if one adopts the WW95 Fe yields as functions of metallicity they tend to overproduce Fe which then needs to be lowered by a factor of $\sim 2$. Concerning the
Fe yields from N97 and LC03, in both cases Fe needs to be slightly increased in the range 13-25 $M_{\odot}$ (LC03) and in the range $18-35 M_{\odot}(\mathrm{N} 97)$. We recall here that Fe and Fe-peak elements ( $\mathrm{Sc}, \mathrm{Fe}, \mathrm{Ni}, \mathrm{Co}, \mathrm{Zn}$ ) are strongly dependent on the chosen mass cut, and different yields for these elements can be obtained under different assumptions on the mass-cut (although not independently), as shown by Nakamura et al. (1999).

The yields of Mn should be increased only in the range 13-18 $M_{\odot}$ and decreased for stars $>30 M_{\odot}$ by a factor of $\sim 2.5$ relative to the WW95 yields. For the massive star calculations of N97, Mn and Cr should be decreased for masses $M \geq 25 M_{\odot}$ whereas Sc should be increased by huge factors in the range 13-25 $M_{\odot}$.

By examining Figs. 7-9 we can conclude that the yields of WW95 need less correction of the number of elements, whereas the yields of N97 need corrections for all the studied isotopes as well as those of LC03. However, the yields of LC03 generally need relatively small corrections with the exception of $\mathrm{K}$ and $\mathrm{Ni}$. Concerning the yields from type Ia SNe (Iwamoto et al. 1999, model W7), those which need a revision are $\mathrm{Mg}$ (should be higher), Ti (higher), Sc (almost a factor of 100 higher), Zn (higher), Co (higher), K (lower) and $\mathrm{Ni}$ (lower). The nucleosynthesis of $\mathrm{Zn}$ has already been studied by Matteucci et al. (1993) who concluded that the $\mathrm{Zn}$ yields from type Ia $\mathrm{SNe}$ should be higher by a factor of $\sim 10$ relative to model W7 of Nomoto et al. (1984).

\section{Summary and conclusions}

In this paper we compared theoretical predictions about the $[\mathrm{el} / \mathrm{Fe}]$ vs. $[\mathrm{Fe} / \mathrm{H}]$ trends in the solar neighborhood for several chemical elements $(\mathrm{O}, \mathrm{Mg}, \mathrm{Si}, \mathrm{Ca}, \mathrm{K}, \mathrm{Ti}, \mathrm{Sc}, \mathrm{Ni}, \mathrm{Mn}, \mathrm{Co}, \mathrm{Fe}$ and $\mathrm{Zn}$ ) with high quality spectroscopic data. In particular, we considered the very recent abundance determinations by Cayrel et al. (2004) in the metallicity range $[\mathrm{Fe} / \mathrm{H}]=-3.0$ down to -4.0 dex. These data allow us to impose constraints both on the element production over the whole galactic lifetime and on all masses contributing to chemical enrichment. They allow us to suggest precise yield ratios for stars up to $\sim 35 M_{\odot}$, since for the more massive ones we would need observed abundance ratios for $[\mathrm{Fe} / \mathrm{H}]<-4.0$. For metallicities between $[\mathrm{Fe} / \mathrm{H}]=-3.0$ and -4.0 stars with masses between $\sim 30$ and $35 M_{\odot}$ contribute to the Galactic chemical enrichment. The comparisons performed in this paper allow us also to infer constraints on the nucleosynthesis in type Ia SNe since we have considered the behavior of the $[\mathrm{el} / \mathrm{Fe}]$ ratios over the whole $[\mathrm{Fe} / \mathrm{H}]$ range.

Our main conclusions can be summarized as follows:

- A homogeneous model for the early halo chemical evolution is able to fit the observations, for metallicities $[\mathrm{Fe} / \mathrm{H}]<$ -3.0 dex, since the data for $[\mathrm{el} / \mathrm{Fe}]$ at these low metallicities show a small spread (typically $0.2-0.3 \mathrm{dex}$ ).

- The two-infall model for the chemical evolution of the Milky Way (Chiappini et al. 1997; Chiappini et al. 2001), which relaxes the instantaneous recycling approximation but retains the instantaneous mixing approximation, can provide an excellent fit to the majority of the data in the Milky Way and in particular to the relative abundance 


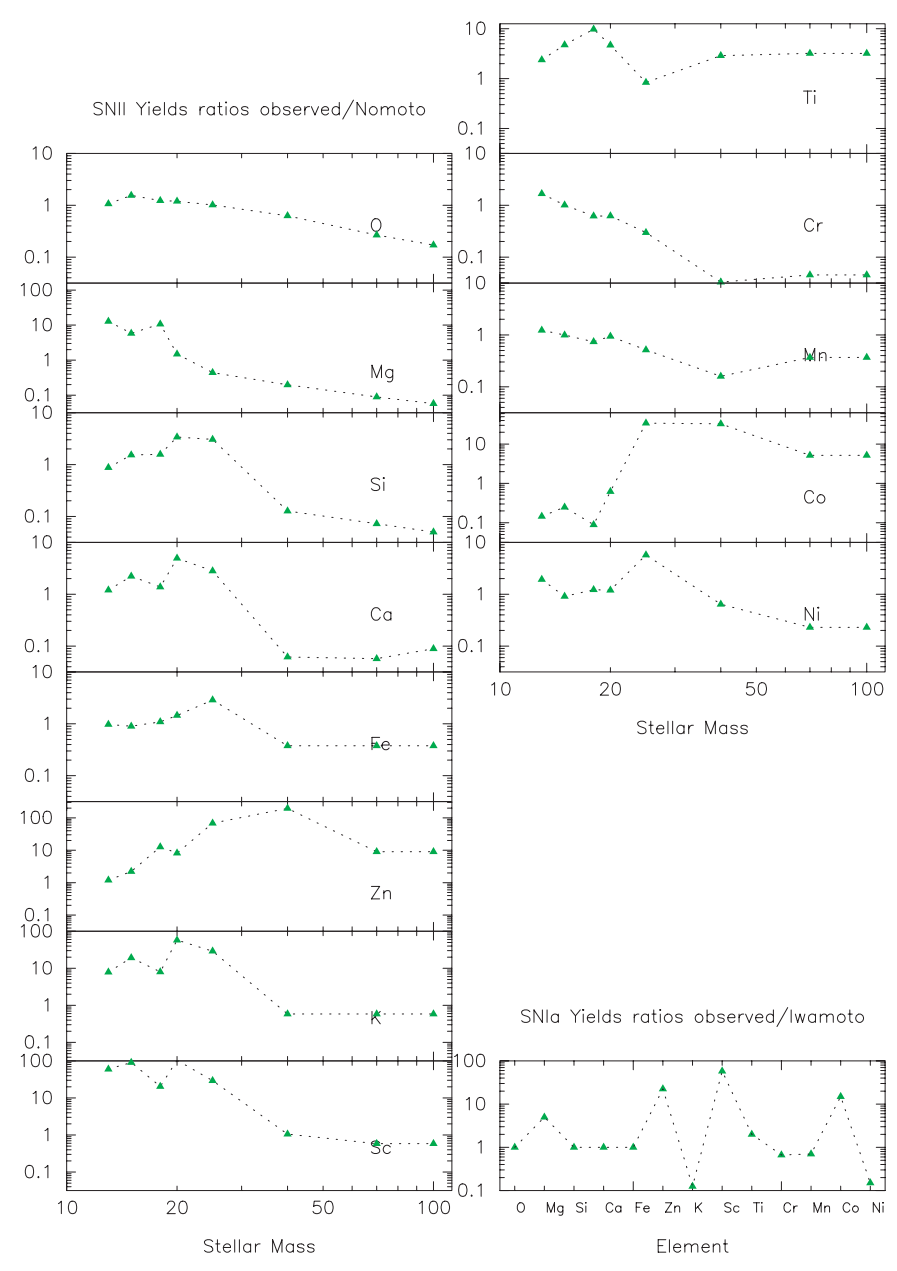

Fig. 8. Ratios between the yields we adopted to obtain the best fit to the data and the yields of N97 for massive stars and those of Iwamoto et al. (1999) for type Ia SNe.

ratios in the whole metallicity range (from $[\mathrm{Fe} / \mathrm{H}]=-4.0$ to 0$)$.

- The most important factor in reproducing the [el/Fe] vs. $[\mathrm{Fe} / \mathrm{H}]$ relations as well as the solar absolute abundances in the solar neighborhood is the combination of the yields from single low and intermediate mass stars, type Ia and II supernovae. We adopted the star formation and infall laws that best reproduce the majority of the observations in the Milky Way, which include other and independent constraints, such as the G-dwarf metallicity distribution, the age-metallicity relation, the current amount of gas and fraction of stars, the current star formation rate and infall rate as well as the current $\mathrm{SN}$ rates and their ratio. In other words, we are not allowed to change the star formation rate history, the infall rate history and the IMF since they have been tested already on a large amount of observational data.

- Here, we discuss only elements for which the contribution of type Ia and II SNe is relevant. Concerning the yields of SN II we find that the yields of WW95 provide the best fit: in particular, no modification is required for the yields of $\mathrm{Ca}, \mathrm{Fe}, \mathrm{Zn}$, and $\mathrm{Ni}$ as computed for a solar chemical composition. For oxygen, the best fit is given by the WW95 yields computed as functions of

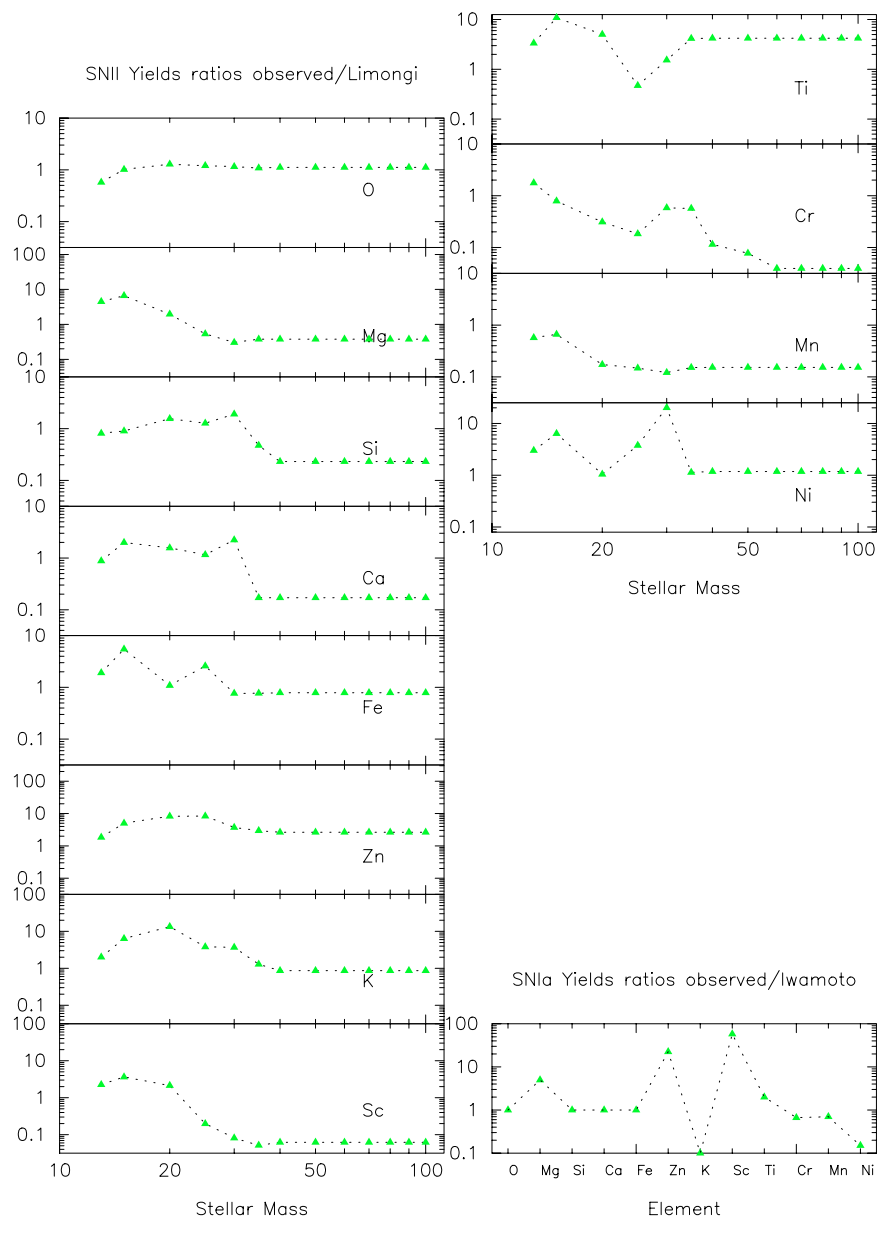

Fig. 9. Ratios between the yields we adopted to obtain the best fit to the data and the yields of LC03 for massive stars and those of Iwamoto et al. (1999) for the nucleosynthesis in type Ia SNe.

metallicity. For the other examined elements $(\mathrm{Mg}, \mathrm{Si}, \mathrm{Ti}$, $\mathrm{K}, \mathrm{Sc}, \mathrm{Co}, \mathrm{Cr}$ and $\mathrm{Mn}$ ), variations of various amounts in the predicted yields are required. These results are rather robust as we ran numerous models spanning a wide range of yields both for SNIa and SNII. However, the numbers shown in Tables 1 and 2 have to be taken more as median values than definitive values, the aim of this paper beeing to pinpoint in which mass range the yields need revision. Another important point shown in Figs. 7 to 9 concerns the relative contributions of SNIa and SNII to the enrichment of the Galaxy. While uncertainties are expected in the predicted yields of the Fe-peak elements mainly related to the mass-cut (Sc, $\mathrm{Cr}, \mathrm{Mn}, \mathrm{Co}$ ), for the $\alpha$-elements $(\mathrm{Mg}, \mathrm{Si}, \mathrm{Ti})$ it is more difficult to envisage why the yields should be different. In particular, a common feature, relative to $\mathrm{Mg}$ yields in massive stars as computed by different authors, is the need to substantially increase the produced and ejected $\mathrm{Mg}$ in stars between 11 and $20 M_{\odot}$ and to decrease it for larger masses. Probably a lower value of the rate of the ${ }^{12} \mathrm{C}(\alpha, \gamma){ }^{16} \mathrm{O}$ reaction could help in increasing the $\mathrm{Mg}$ yields but it would affect the yields of $\mathrm{Si}$ and $\mathrm{Ca}$ (Limongi, private communication) or a different treatment of convection. For K, the situation is more complicated since there are contributions to this elements also from neutrino-induced reactions. 
- We tested the yields for SN II as computed by N97 and LC03 and also in these cases we conclude that modifications are required to obtain the best fit to the observations. In particular, for the yields of N97, various modifications are required for all the studied elements; for the yields of LC03 the O yields should be almost untouched.

- For the yields from type Ia $\mathrm{SNe}$, a revision is needed for $\mathrm{Mg}$, Ti, Sc, K, Co, Ni and $\mathrm{Zn}$. In particular, while values of $\mathrm{Mg}, \mathrm{Ti}, \mathrm{Sc}, \mathrm{Zn}$ and $\mathrm{Co}$ should be larger, those of $\mathrm{K}$ and $\mathrm{Ni}$ should be smaller than predicted. Whether these proposed modifications are physically plausible is still to be assessed by the experts in the nucleosynthesis field.

Acknowledgements. We would like to thank Marco Limongi for very useful comments on the nucleosynthesis aspects. F.M and C.C. acknowledge financial support from INAF (Italian national institute for astrophysics) contract n.2003028039. P.F. and C.C. acknowledge support under the ESO visitor program in Garching during the completion of a part of this work.

\section{References}

Alibés, A., Labay, J., \& Canal, R. 2001, A\&A, 370, 1103

Allende-Prieto, C., Lambert, D. L., \& Asplund, M. 2001, ApJ, 556, L63

Argast, D., Samland, M., Thielemann, F.-K., \& Gerhard, O. E. 2002, A\&A, 388, 842

Audouze, J., \& Silk, J. 1995, ApJ, 451, L49

Beers, T. C., Preston, G. W., \& Schectman, S. A. 1992, AJ, 102, 1987

Beers, T. C., Beers, R. S., Norris, J. E., Ryan, S. G., \& Shefler, T. 1999, AJ, 117, 981

Chang, R. X., Hou, J. L., Shu, C. G., \& Fu, C. Q. 1999, A\&A, 350, 38

Cappellaro, E., Evans, R., \& Turatto, M. 1999, A\&A, 351, 459

Carney, B. W., Wright, J. S., Sneden, C., et al. 1997, AJ, 114, 363

Carretta, E., Gratton, R. G., Cohen, J. G., et al. 2002, AJ, 124, 481

Cayrel, R., Depagne, E., Spite, M., et al. 2004, A\&A, 416, 1117

Chiappini, C., Matteucci, F., \& Gratton, R. 1997, ApJ, 477, 765

Chiappini, C., Matteucci, F., \& Romano, D. 2001, ApJ, 554, 1044

Chiappini, C., Matteucci, F., Beers, T. C., \& Nomoto, K. 1999, ApJ, 515,226

Edvardsson, B., Andersen, J., Gustafsson, B., et al. 1993, A\&A, 275, 101

Fulbright, J. P. 2000, AJ, 120, 1841

Furhmann, K. 1998, A\&A, 338, 161

Gilroy, K. K., Sneden, C., Pilachowski, C. A., \& Cowan, J. J. 1988, ApJ, 327, 298
Goswami, A., \& Prantzos, N. 2000, A\&A, 359, 191

Gratton, R. G., \& Sneden, C. 1988, A\&A, 204, 193

Gratton, R., \& Sneden, C. 1994, A\&A, 287, 927

Gratton, R., Carretta, E., Matteucci, F., \& Sneden, C. 2000, A\&A, 358,671

Greggio, L., \& Renzini, A. 1983, A\&A, 118, 217

Grevesse, N., \& Sauval, A. J. 1998, Space Sci. Rev., 85, 161

Gerritsen, J. P. E., \& Icke, V. 1997, A\&A, 325, 972

Imbriani, G., Limongi, M., Gialanella, L., et al. 2001, ApJ, 558, 903

Iwamoto, K., Brachwitz, F., Nomoto, K., et al. 1999, ApJS, 125, 439

Kennicutt, R. C. Jr. 1989, ApJ, 344, 685

Kennicutt, R. C. Jr. 1998, ApJ, 498, 541

Limongi, M., \& Chieffi, A. 2003, ApJ, 592, 404

Madau, P., della Valle, M., \& Panagia, N. 1998, MNRAS, 297, 17

Martin, C. L., \& Kennicutt, R. C. Jr. 2001, ApJ, 555, 301

Matteucci, F., \& Francois, P. 1989, MNRAS, 239, 885

Matteucci, F., \& Greggio, L. 1986, A\&A, 154, 279

Matteucci, F., \& Recchi, S. 2001, ApJ, 558, 351

Matteucci, F., Raiteri, C. M., Busson, M., Gallino, R., \& Gratton, R. 1993, A\&A, 272, 421

Matteucci, F. 2001, The Chemical Evolution of the Galaxy, ASSL (Kluwer Academic Publishers)

McWilliam, A., Preston, G. W., Sneden, C., \& Searle, L. 1995, AJ, 109,2757

Nakamura, T., Umeda, H., Nomoto, K., Thielemann, F.-K., \& Burrows, A. 1999, ApJ, 517, 193

Nissen, P. E., \& Schuster, W. J. 1997, A\&A, 326, 751

Nissen, P. E., Primas, F., Asplund, M., \& Lambert, D. L. 2002, A\&A, 390,235

Nomoto, K., Thielemann, F.-K., \& Yokoi, K. 1984, ApJ, 286, 644

Nomoto, K., Iwamoto, N., Nakasato, N., et al. 1997, Nucl. Phys. A, 621,467

Norris, J. E., Ryan, S. G., \& Beers, T. C. 2001, ApJ, 561, 1034

Pagel, B. E. J., \& Tautvaisiene, G. 1995, MNRAS, 276, 505

Portinari, L., \& Chiosi, C. 1999, A\&A, 350, 827

Rauscher, T., Hoffman, R. D., \& Woosley, S. E. 2002, ApJ, 576, 323

Reid, M. J. 1993, ARA\&A, 31, 345

Ryan, S., Norris, J. E., \& Bessell, M. S. 1991, AJ, 102, 303

Ryan, G., Norris, E., \& Beers, C. 1996, ApJ, 471, 254

Scalo, J. M. 1986, FCPh, 11, 1

Stephens, A. 1999, AJ, 117, 1771

Thomas, D., Greggio, L., \& Bender, R. 1998, A\&A, 296, 119

Travaglio, C., Galli, D., Gallino, R., et al. 1999, ApJ, 521, 691

Umeda, H., \& Nomoto, K. 2002, ApJ, 565, 385

van den Hoek, L. B., \& Groenewegen, M. A. T. 1997, A\&AS, 123, 305

Woosley, S. E., \& Weaver, T. A. 1995, ApJS, 101, 181 